\title{
Complete Agenesis of Both Metatarsophalangeal Sesamoids with Contralateral Agenesis of Fibular Sesamoid Associated with Hallux Valgus
}

\author{
by Lyndon W. Mason, MB BCh MRCS (Eng) ${ }^{1} \otimes$, Gemma Digby, MB BCh$^{2} \otimes$, \\ Hiro Tanaka, MB BCh MSc (Orth Eng) FRCS (Ed) FRCS (Orth) ${ }^{3}$ 四
}

The Foot and Ankle Online Journal 5 (4): 2

We report a rare case of complete hallucal sesamoid agenesis in combination with severe symptomatic hallux valgus. This was successfully treated with a Scarf osteotomy. We present 2 case illustrations of hallux valgus deformity with sesamoid agenesis to contribute to the literature.

Keywords: Complete Sesamoid Agenesis, Metatarsalgia, Fibular Sesamoid Agenesis.

Accepted: March, $2012 \quad$ Published: April, 2012

This is an Open Access article distributed under the terms of the Creative Commons Attribution License. It permits unrestricted use, distribution, and reproduction in any medium, provided the original work is properly cited. @The Foot and Ankle Online Journal (www.faoj.org), 2011 All rights reserved.

$\mathrm{T}$ The metatarsophalangeal joint of the great toe differs from that of the lesser toes because it has a sesamoid mechanism and a set of intrinsic muscles that stabilize the joint and provide motor strength to the first ray. ${ }^{1}$ The hallucal sesamoids are a consistent entity in humans, originating in the tendons of flexor hallucis brevis. They appear within the seventh or eighth week of embryonic development as islands of undifferentiated connective tissue.

Address correspondence to: Mr Lyndon Mason, Address correspondence to: Foot and Ankle Unit, Trauma and Orthopaedic Department, Royal Gwent Hospital, Newport, NP20 2UB

E-mail: mrlyndonmason@me.com

1,2,3 Foot and Ankle Unit, Trauma and Orthopaedic Department, Royal Gwent Hospital, Newport, NP20 2UB
By the twelfth week, chondrification begins, but it is not until 8 to 10 years of life through multiple ossification centers that they ossify. ${ }^{2}$ Often these centers of ossification do not coalesce and remain partite in a significant portion of the population. The function of the hallucal sesamoids is to absorb weight-bearing forces and enhance the load-bearing capacity of the first ray. They power plantar flexion of the first ray by increasing the moment of the flexor hallucis brevis and function to elevate the first metatarsal head, which dissipates the forces on the metatarsal head. ${ }^{3}$ 


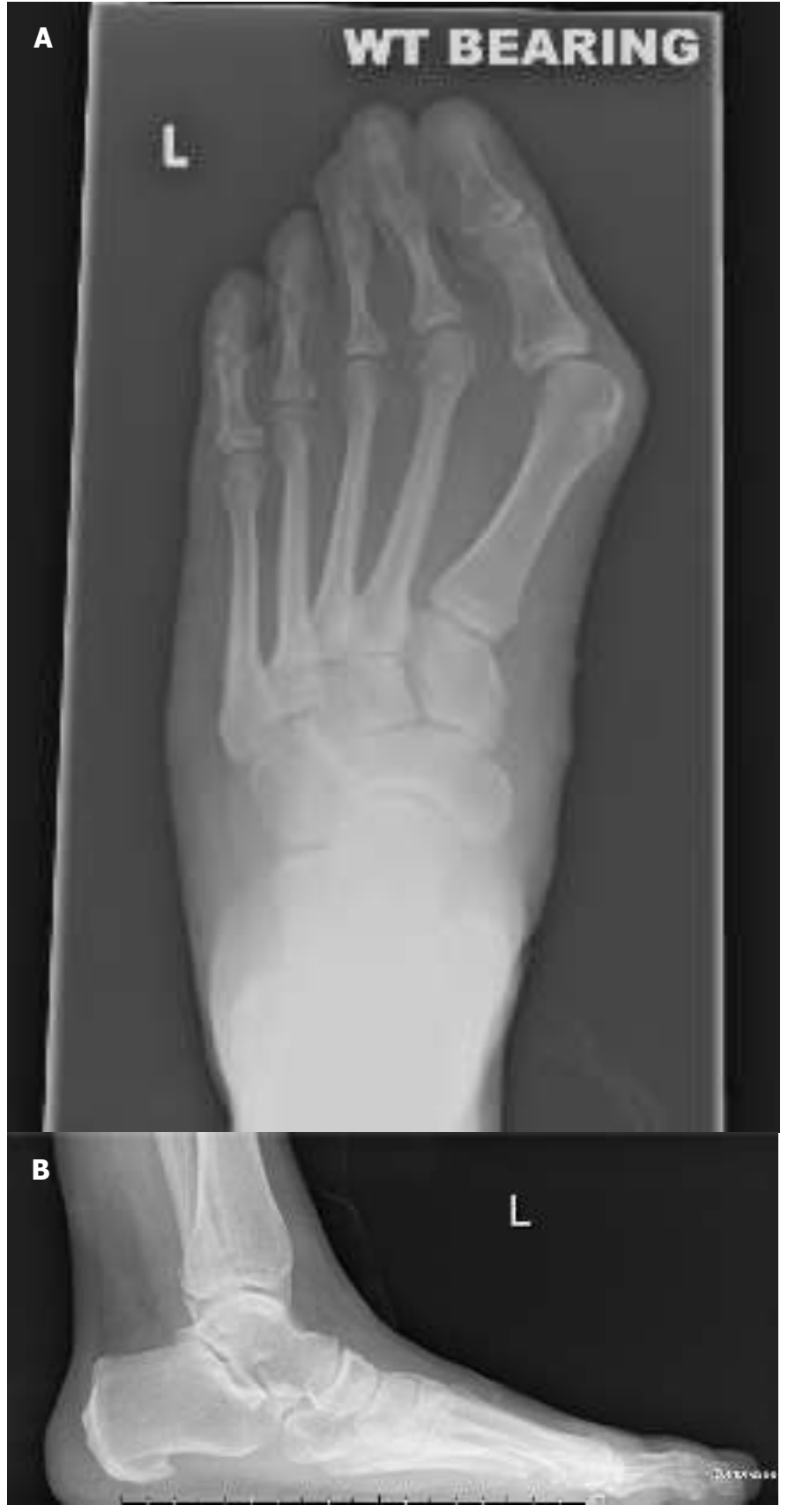

Figure 1A and 1B Left foot weight bearing anteroposterior (A) and lateral (B) radiographs illustrating hallux valgus and complete lack of hallucal sesamoids.

On a review of the literature, Le Minor found that in 3305 feet there were no reports of sesamoid absence. ${ }^{4}$ There are eleven case reports of single sesamoid agenesis in the literature, seven cases of which are tibial sesamoid agenesis ${ }^{5-10}$ and three cases of fibular sesamoid agenesis. ${ }^{11-13}$

(C) The Foot and Ankle Online Journal, 2012

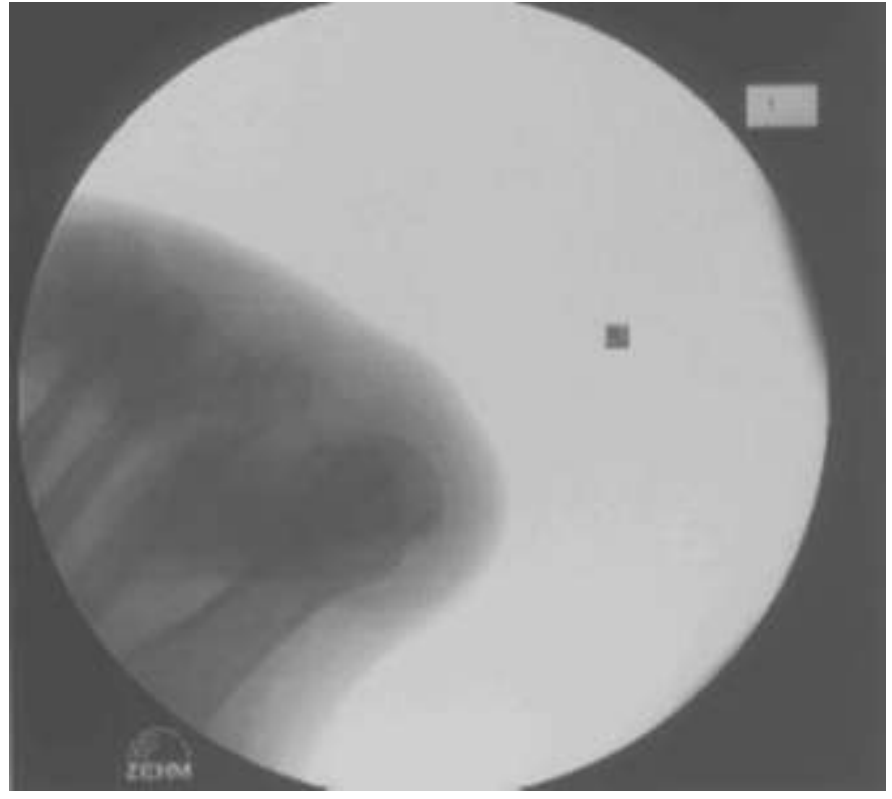

Figure 2 Intraoperative sesamoid view of the left foot showing lack of hallucal sesamoids, and no discernible sesamoid grooves or crista.

There is one reported case of unilateral complete agenesis of the sesamoids ${ }^{14}$ and one bilateral complete agenesis of the sesamoids reported in the literature. ${ }^{15}$ More recently, there was a report of adolescence hallux valgus with bilateral absence of hallux sesamoids. ${ }^{16}$ We report a case of unilateral complete absence of sesamoids with contralateral agenesis of the fibular sesamoid with symptomatic hallux valgus.

\section{Case Report}

A 49 year-old, Caucasian male, who worked as a line manager in a hospital laundry department, attended our clinic due to a painful left hallux valgus. He experienced pain over the medial eminence of his left great toe due to rubbing shoe wear, and on the plantar surface of his $1^{\text {st }}$ metatarsophalangeal joint after exertion. Pain and stiffness of the left first metatarsophalangeal joint was also experienced in the morning. He enjoyed evening walks and found this was being curtailed due to pain. He was a type 2 diabetic patient being treated with an oral hyperglycemic medication. 


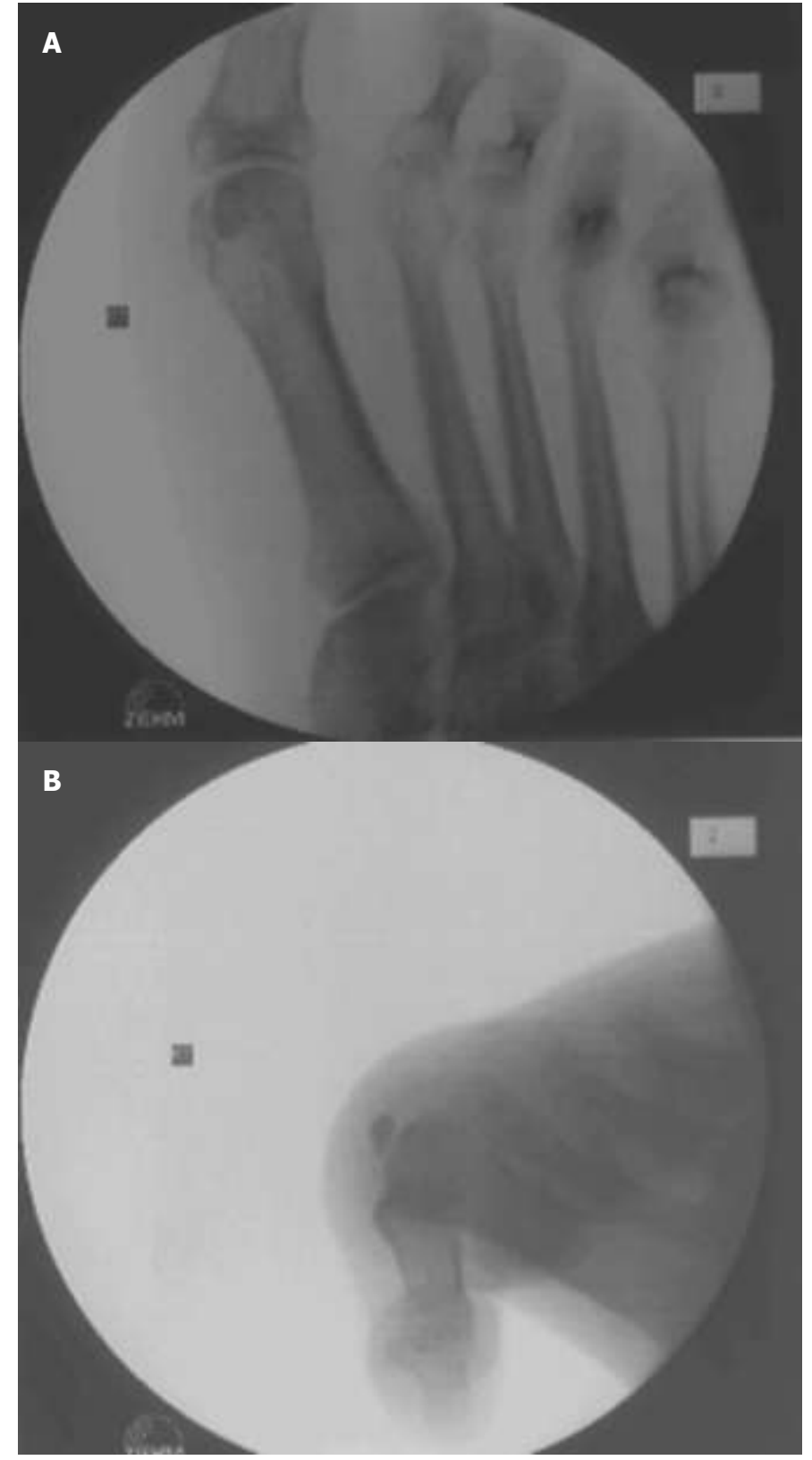

Figure 3A and 3B Right foot anteroposterior radiograph (A) and sesamoid view (B) showing tibial sesamoid presence and absence of fibular sesamoid, with no deformity at the first metatarsophalangeal joint.

On examination it was noted that he had bilateral severe hallux valgus with callosities under both first metatarsophalangeal joints. The left first metatarsophalangeal joints had a pain free range of motion of $30^{\circ}$ dorsiflexion to $50^{\circ}$ plantarflexion.
Radiographs illustrated a left forefoot adductus with severe hallux valgus (Inter-Metatarsal Angle $16^{\circ}$ and Hallux Valgus Angle of $25^{\circ}$ ), and it was noted a complete absence of sesamoids. (Figures 1A, 1B and 2) The patient had normal sensation and a normal vascular exam.

The left symptomatic hallux valgus was treated with surgical intervention. We performed a lateral release through a small dorsal incision and a medial eminence resection with Scarf osteotomy through a mid medial incision as described by Barouk. ${ }^{17}$ Intraoperatively, there was no evidence of tibial or fibular sesamoids. Intraoperative radiographs of the contralateral foot revealed absence of the fibular sesamoid but presence of the tibial sesamoid. This foot had no discernible hallux valgus deformity. (Figures $3 \mathrm{~A}$ and $3 \mathrm{~B}$ ) Post operatively, the patient was mobilized in a heel weight bearing shoe for 6 weeks and radiographs obtained at this stage showed satisfactory reduction in the intermetatarsal angle (Figures 4A and 4B). The patient's symptoms were successfully treated with this procedure, and remains recurrence free at 3 years postoperative.

\section{Discussion}

Not much is known of the repercussions of sesamoid agenesis. Most of the single sesamoid agenesis in the literature were reported to be asymptomatic, apart from one case of associated metatarsalgia. Metatarsalgia was also the complaint of the only reported case of unilateral complete sesamoid agenesis. ${ }^{15}$ The bilateral complete sesamoid agenesis reported hallux varus deformity, however there has been no associated hallux valgus deformity reported with sesamoid agenesis. ${ }^{14}$ In comparison, the acquired loss of hallucal sesamoids through surgery or injury can result in significant deformity. Removal of the fibular sesamoid as described in the McBride procedure for hallux valgus, can result in hallux varus. $^{18}$ The loss of both sesamoids during hallux valgus surgery or through injury has been reported to cause hallux malleolus, hallux varus or hallux valgus. $18-21$ 


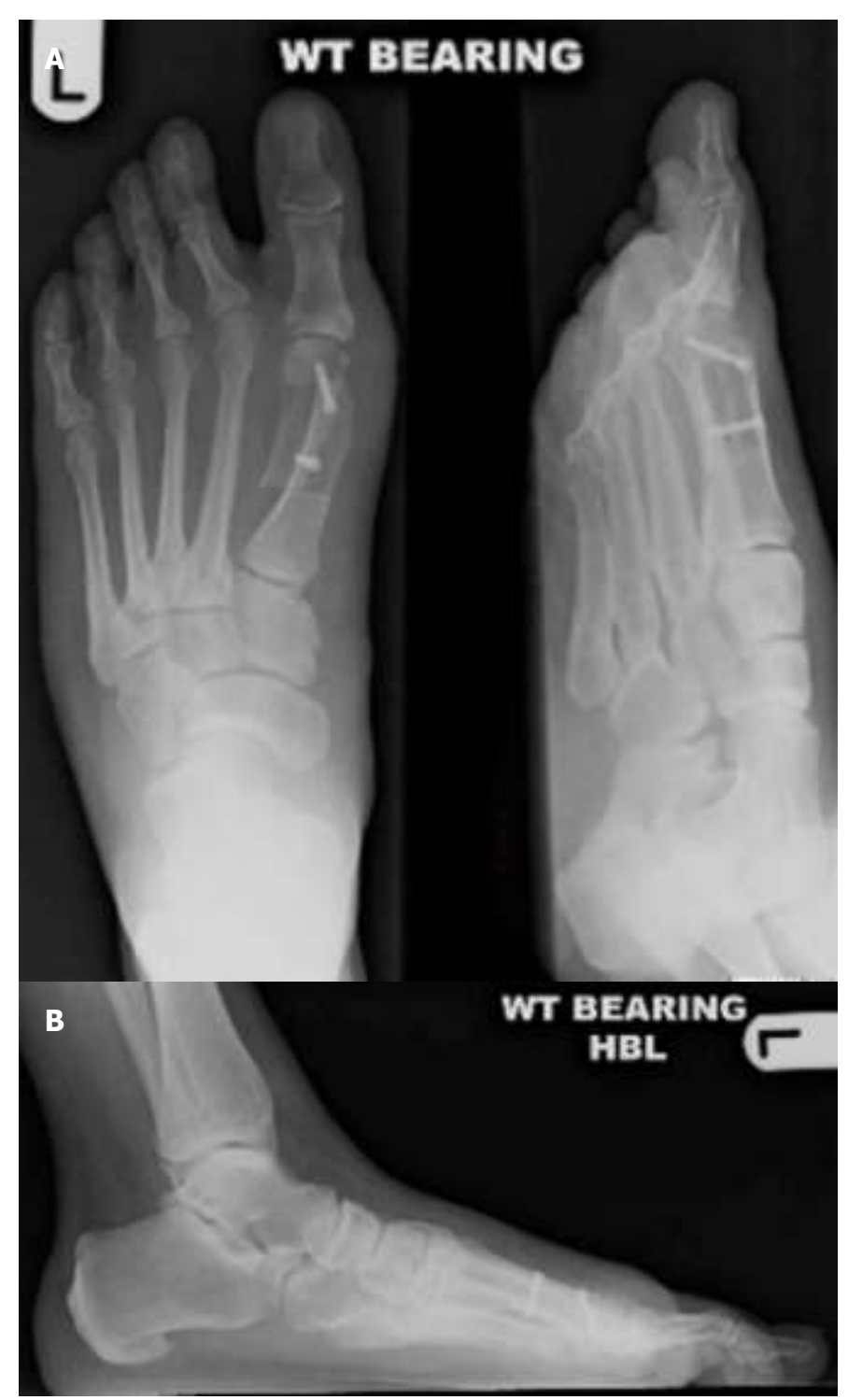

Figure 4A and 4B Anteroposterior (A) and lateral (B) weight bearing radiographs post Scarf osteotomy showing correction of intermetatarsal angle and hallux valgus.

However, total sesamoidectomy for painful hallux rigidus has been reported to not cause such complications. ${ }^{22}$ It is unlikely that the sesamoid itself but rather the surrounding ligamentous structures that are insufficient in causing the deformities present in surgical excision of the sesamoid, and possibly why it has not been reported in any cases of sesamoid agenesis, either partial or complete, until now.
It is generally accepted that the medial supporting structures of the first metatarsophalangeal joint, i.e. the metatarsosesamoid ligament, phalangealsesamoid and medial collateral ligaments, fail early in hallux valgus deformity. ${ }^{23}$ The proximal phalanx that is anchored at its base to the sesamoids by the plantar plate is pulled into valgus and pronation. It is important to note that the oblique head of the adductor hallucis muscle inserts onto the lateral sesamoid and the lateral capsule of the metatarsophalangeal joint and the transverse head inserts onto the lateral sesamoid, lateral capsule of the metatarsophalangeal joint and the lateral plantar side of the proximal phalanx..$^{23,24}$ As the medial sesamoid ligament is one of the key structures in the valgus stability of the first metatarsophalangeal joint, it is likely in this case to have been weaker as a consequence of sesamoid agenesis.

We treated this patient with a Scarf osteotomy which resulted in a successful outcome. This does not address the anatomical insufficiencies, but by shifting the metatarsal head laterally, the soft tissues are once again balanced with the flexor and extensor hallucis longus no longer providing deforming forces. This is only anecdotal, but considering the rarity of this case we would recommend a first metatarsal osteotomy for the treatment of such a case in the future. The patient's symptoms were a consequence of the hallux valgus deformity and not the absence of sesamoids. However, it is likely that the sesamoid absence would have predisposed the foot to the development of hallux valgus.

\section{References}

1. Stein HC. Hallux Valgus. Surg Gynec Obstet 1938 66: 889898.

2. Sammarco GJ, Idusuyi OB. Complications after surgery of the hallux. Clin Orthop Relat Res 2001 391: 59-71. [PubMed] 3. Aper RL, Saltzman CL, Brown T. The effect of hallux sesamoid excision on the flexor hallucis longus moment arm. Clin Orthop Relat Res 1996 325: 209-217. [PubMed] 4. Le Minor JM. Congenital absence of the lateral metatarsophalangeal sesamoid bone of the human hallux: a case report. Surg Radiol Anat 1999 21(3): 225-227. [PubMed] 5. Kanatli U, Ozturk AM, Ercan NG, Ozalay M, Daglar B, Yetkin $\mathrm{H}$. Absence of the medial sesamoid bone associated with metatarsophalangeal pain. Clin Anat 2006 19: 634639. [PubMed] 
6. Day F, Jones PC, Gilbert CL. Congenital absence of the tibial sesamoid. JAPMA 2002 92:153-154. PubMed]

7. Jeng CL, Maurer A, Mizel MS. Congenital absence of the hallux fibular sesamoid: a case report and review of the literature. Foot Ankle Int 1998 19: 329-331. [PubMed]

8. Goez J, DeLauro T. Congenital absence of the tibial sesamoid. JAPMA 1995 85: 509-510. [PubMed]

9. Zinsmeister BJ, Edelman R. Congenital absence of the tibial sesamoid: a report of two cases. J Foot Surg. 1985 24: 266-268.

10. Inge GA. Congenital absence of the medial sesamoid of the great toe. JBJS 1936 18A: 188-190.[PubMed]

11. Williams TH, Pasapula C, Robinson AH. Complete sesamoid agenesis: a rare cause of first ray metatarsalgia. Foot Ankle Int 2009 30: 465-467.[PubMed]

12. Yildirim Y, Saygi B. Congenital absence of the lateral sesamoid. JAPMA 2006 96:78-81. [PubMed]

13. Lapidus PW. Congenital unilateral absence of the medial sesamoid of the great toe: Report of a case. JBJS 1939 21A: 208209. [WebSite]

14. Wilson D. Treatment of hallux valgus and bunions. Br J Hosp Med 1980 24: 548-559. [PubMed]

15. Wright SM. Congenital hallux varus deformity with bilateral absence of the hallucal sesamoids. JAPMA 1998 88: 47-48.

PubMed]

16. Alshryda S, Lou T, Faulconer ER, Adedapo AO., Adolescent hallux valgus deformity with bilateral absence of the hallucal sesamoids: a case report. J Foot Ankle Surg 2012 51: 80-82. [Pub Med]

17. Barouk LS. Scarf osteotomy of the first metatarsal in the treatment of hallux valgus. Foot Diseases 1995 2: 35-48.

18. Cohen BE. Hallux sesamoid disorders. Foot Ankle Clin Mar 2009 14: 91-104. [PubMed]

19. Grace DL. Sesamoid problems. Foot Ankle Clin 2000 5: 609-627. [PubMed]

20. Campbell AC, McBride DJ, Anderso EG. Surgical treatment in disorders of the sesamoids of flexor hallucis brevis. The Foot 1993 3: 43-45. [Website]

21. Richardson EG. Injuries to the hallucal sesamoids in the athlete. Foot Ankle 1987 7: 229-244. PubMed]

22. Tagoe M, Brown HA, Rees SM. Total sesamoidectomy for painful hallux rigidus: a medium-term outcome study. Foot Ankle Int 2009 30: 640-646. [PubMed]

23. Arakawa T, Tokita K, Miki A, Terashima T. Anatomical study of human adductor hallucis muscle with respect to its origin and insertion. Ann Anat 2003185: 585-592. [PubMed] 24. Perera AM, Mason L, Stephens MM. Current concepts review: The pathogenesis of hallux valgus. JBJS 2011 93A:16501661. PubMed] 